\title{
CARACTERISTICAS DE LAS CUOTAS DE MER- CADO, DE LOS INDICES DE RIQUEZA ACTIVA Y DE LOS NIVELES DE DESARROLLO DE LAS ZONAS Y AREAS COMERCIALES
}

por

Ignacio Ballester Ros

SUMARIO: I. INTRODUCCION.-II. LA CUOTA DE MERCADO DE LAS ZONAS Y AREAS COMERCIALES EN 1979 Y SU EVOLUCION EN EL PERIODO 1967-1979: 1. LA CUOTA DE MERCADO EN LAS ZoNaS Y AREAS COMERCIALES EN 1979. 2. LA EVOLUCIÓN DE LA CUOTA DE MERCADO EN LAS ZONAS Y AREAS COMERCIALES EN EL PERIODO 1967-1979. 3. LA CUOTA DE MERCADO PER CÁPITA Y SU EVOLUCIÓN.-III. LA CUOTA DE RIQUEZA ACTIVA DE LAS ZONAS Y DE LAS AREAS COMERCIALES: 1. EL INDICE DEMOGRAFICO. 2. El ÍNDICE CULTURAL. 3. El ÍNDICE ECONÓMICO. 4. La CUOTA DE RIQUEZA ACTIVA.-IV. EL INDICE TURISTICO Y EL NIVEL DE DESARROLLO.-V. APENDICE ESTADISTICO: 1. EvOLUCIÓN DE LAS CUOTAS DE MERCADO EN LAS ZONAS Y EN LAS AREAS COMERCIALES DURANTE EL PERIODO 1967-1979. 2. LOS ÍNDICES DEMOGRAFICO, CULTURAL Y ECONOMICO Y LA CUOTA DE RIQUEZA ACTIVA EN LAS ZONAS Y EN LAS AREAS COMERCIALES.

\section{INTRODUCCION}

Una vez más, acaba de aparecer, en su última edición, el Anuario del Mercado Español, correspondiente al año 1981, que patrocina y edita el Banco Español de Crédito. 
Su contenido es el tradicional. Una primera parte dedicada a la geografía del mercado, que este año comprende, de una parte, la investigación, ya clásica, sobre las cuotas de mercado de las Zonas y de las Areas Comerciales de España, y de otra, el trabajo extraordinario a que dedica su primordial atención el equipo de estadísticos y economistas que elabora el Anuario, trabajo de estudio e investigación que versa sobre «España ante la ampliación de la Comunidad Económica Europea», que contiene un análisis sectorial, comparativo de España con cada uno de los países de la Comunidad, respecto a la estructura y evolución demográfica, rentas y finanzas, agricultura, ganadería y pesca, energía, industria por sectores, transportes, comercio exterior, turismo e indicadores económicos y sociales y una síntesis del nivel de vida.

Sigue una segunda parte dedicada al estudio de las fuentes de riqueza e instrumentos del mercado-consumos energéticos, índices de capacidad de compra provincial y equipamiento comercial-. En la tercera parte se trata de los índices de riqueza activa de las Areas Comerciales. En la cuarta se abordan los indicadores socioeconómicos del mercado español. En la quinta se analiza la coyuntura y se fijan los índices de vivacidad y los movimientos migratorios interiores. En la sexta y última se presenta la información estadística referida a cada uno de los Municipios españoles con población superior a 3.000 habitantes.

Dada la amplitud y variedad de temas a que se refiere la información que contiene el Anuario, y la imposibilidad de abordarlos todos en un solo trabajo, limitado en su extensión, así como la próxima publicación de los resultados del Censo de la Población de España, con referencia al mes de marzo de este año, que nos ha de permitir el conocimiento de la cifra de habitantes de cada uno de los Municipios españoles, parece más oportuno dedicar este comentario al análisis de las cuotas de mercado de las Zonas y Areas Comerciales en 1979, y a su evolución en el período 1967 a 1979, y a las cuotas de riqueza activa y niveles de desarrollo en 1979, investigaciones que sistemáticamente se abordan cada dos años por el equipo que elabora el Anuario.

Estas investigaciones revisten excepcional importancia por cuanto facilitan el conocimiento de la estructura demográfica, cultural y económica de las 52 Zonas y 101 Areas Comerciales ya configuradas en el Atlas del Mercado Español, su distinto peso y evolución 
con arreglo a las últimas cifras actualizadas, la cuota de riqueza que corresponde a cada una y las perspectivas del nivel de desarrollo operado en los últimos años.

\section{LA CUOTA DE MERCADO DE LAS ZONAS Y AREAS COMERCIALES EN 1979 Y SU EVOLUCION EN EL PERIODO 1967-1979}

\section{La CUOTA de mercado de las Zonas y AREAS Comerciales en 1979}

Tomando como base la población de hecho registrada en el Padrón renovado de Habitantes de 1975, el número de licencias comerciales, de entidades bancarias y de teléfonos-incluidas las solicitudes pendientes de instalación-y la recaudación por el impuesto de espectáculos públicos en el año 1979 , se ha llevado a cabo la más reciente determinación y distribución de la cuota de mercado de las Zonas y Areas Comerciales.

Como ya se sabe, la cuota de mercado es un dato objetivo que indica la cantidad de productos o servicios que, teóricamente y en igualdad de condiciones, pueden absorber las Zonas y Areas Comerciales y expresan comparativamente la efectiva capacidad de compra de los grupos sociales asentados en cada una de ellas.

Se elabora, como en otros bienios, partiendo de los datos individualizados para cada uno de los Municipios españoles-agrupados por Areas-, hallándose el promedio de las proporciones que, en cada serie estadística utilizada, corresponde a cada Area Comercial, sobre una base nacional de 100.000 unidades. Los valores de las Zonas Comerciales se obtienen por meras sumas de los valores de las respectivas Areas.

En la tabla I del Apéndice Estadístico se inserta la cuota de mercado de cada Zona y Area, en los años 1967 y 1979, y la diferencia absoluta entre los valores correspondientes.

En 1979 siguen ocupando los dos primeros lugares las Zonas Comerciales de Madrid y Barcelona-incluidas las Areas de Manresa y Villafranca del Penedés-, con más de 15.000 unidades cada una y escasa diferencia entre ambas, absorbiendo cada una de las dos ciudades una sexta parte de la cuota nacional de mercado y, en conjunto, el 31 por 100. En orden de importancia le siguen, aunque alejadas de ellas por sus valores absolutos, Valencia-con las Areas 
de Gandía, Játiva, Onteniente y Utiel incluidas-, con más de 6.000 unidades, y Bilbao y Sevilla, con más de 3.500 unidades de mercado cada una. Entre las cinco acumulan 44.500 unidades de mercado, el 44,5 por 100 de la cuota de mercado nacional.

Resumiendo los valores de las demás Areas Comerciales, encontramos que ocho de ellas presentan valores entre 2.000 y 2.800 unidades de mercado cada una, y otras 15 ofrecen valores entre 1.000 y 2.000 unidades.

Las 28 Zonas a que nos hemos referido, que tienen, al menos, el 1 por 100 de las unidades de mercado, representan el 85,5 por 100 . de las unidades de mercado, dejando para las 24 Zonas Comerciales restantes tan sólo el 14,5 por 100 de la cuota de mercado nacional.

Las citadas 28 Zonas configuran cuatro grandes Regiones de alta cuota de mercado:

a) Norte, que comprende Galicia-a excepción de Orense-, Asturias, Santander, Vizcaya, Guipúzcoa, Navarra y Zaragoza.

b) Litoral mediterráneo oriental, que abarca Cataluña-excepto Tortosa-, Baleares, Castellón, Valencia, Alicante y Murcia.

c) Central, con Valladolid, Madrid-que figura a la cabezay Badajoz.

d) Sur, que se extiende a toda Andalucía-excepto Almería y Huelva-, y se prolonga hasta Canarias.

En líneas generales, son las Regiones que tantas veces hemos configurado en nuestros comentarios sobre los aspectos demográficos y socioeconómicos de España, marcando las diferencias entre ellas, ya sean Regiones, Zonas o Areas, destacando el matiz discriminatorio entre el centro y la periferia españolas, y la alta concentración del mercado en unas pocas Zonas, las más importantes desde el punto de vista demográfico, con la concentración de la población en grandes urbes, o desde el aspecto económico, en cuanto. concentración industrial o de servicios, en gran parte coincidente con aquélla.

Si en lugar de las Zonas contemplamos a las Areas Comerciales, también los dos primeros lugares corresponden a Madrid y Barcelona, seguidas a cierta distancia de Valencia, Bilbao, Sevilla, Zaragoza y Málaga. Entre las cinco primeras absorben el 42,7 por 100' de la cuota de mercado nacional, y entre las siete, el 47,2, lo que confirma la idea de la alta concentración del mercado en pocas Areas. 
De las restantes Areas sólo 17 presentan una cuota de mercado superior a 1.000 unidades; otras 24 oscilan entre 500 y 1.000 unidades, y 53, más de la mitad de Areas, tienen valores inferiores a 500 unidades de mercado.

Con las 24 Areas con cuota de mercado superior a 1.000 unidades, se mantienen configuradas las mismas cuatro Regiones constituidas con las Zonas Comerciales, si bien más debilitadas. En la Región Norte están las Areas de La Coruña-sin Santiago de Compostela y El Ferrol-, Vigo-sin Pontevedra-, Oviedo-sin Avilés ni Gijón-, Santander, Bilbao, San Sebastián, Pamplona y Zaragoza - sin Calatayud-. En el litoral mediterráneo oriental se hallan las Areas de Lérida-sin Puigcerdá-, Gerona-sin Figueras, Olot ni Vich-, Barcelona-sin Manresa ni Villafranca del Penedés-, Castellón, Valencia-sin Gandía, Játiva, Onteniente y Utiel-, Alicante - sin Alcoy y Elche-, Murcia-sin Cartagena y Lorca-y Palma de Mallorca-sin Ciudadela, Ibiza y Mahón-. En la Región central se mantienen Valladolid y Madrid, esta última a la cabeza de las Areas. En la Región Sur figuran Córdoba, Granada-sin Motril-, Málaga - sin Antequera ni Ronda-y Sevilla, extendiéndose hasta Canarias con las Areas de Santa Cruz de Tenerife y Las Palmas de Gran Canaria.

La consideración, en este orden de cosas, de las Zonas o de las Areas difiere sustancialmente. Mientras que hasta 28 Zonas tienen cuotas de mercado superiores al 1 por 100 , es decir, el 54 por 100 de las Zonas, las Areas con cuota de mercado mayor del 1 por 100 son tan sólo 24, equivalentes al 24 por 100 del total de Areas. Por el contrario, con cuotas de mercado inferiores al 1 por 100 existen el 46 por 100 de las Zonas y el 76 por 100 de las Areas.

\section{LA EVOLUCIÓN DE LA CUOTA DE MERCADO EN LAS ZoNAS y AREAS CoMERciales EN EL PERfodo 1967-1979}

Hemos considerado de utilidad determinar la evolución de la cuota de mercado en las Zonas y Areas Comerciales en dos momentos extremos de las series bienales elaboradas y publicadas en el Anuario del Mercado Español, concretamente entre 1967 y 1979. Las cifras correspondientes figuran en la tabla I del Apéndice.

De su examen se deduce que se ha visto incrementada la cuota de mercado de 22 Zonas Comerciales en el período de referencia, y que, por el contrario, ha disminuido la cuota de mercado de 30 Zonas. 
Las primeras configuran las siguientes Regiones de incremento de cuota de mercado: en el Norte, Galicia, con Asturias, Santander y Vitoria; en el Mediterráneo, Cataluña-excepto Tortosa-, Baleares y la Región valenciana con sus tres Provincias; en el centro, Valladolid y Madrid, y en el Sur, Almería, Málaga, Huelva y Canarias.

Por el contrario, las 30 Zonas que han visto disminuidas sus. cuotas de mercado se agrupan en esta forma: en el Norte, el País Vasco-excepto Vitoria-, Logroño, Navarra y Aragón; en la Meseta, el Valle del Duero-excepto Valladolid-y la Submeseta Sur -excepto Madrid-, y al Sur; Andalucía-menos las tres Zonas citadas en el párrafo anterior-y Murcia.

Por sus incrementos durante el período de referencia destacan. Alicante, Barcelona, Palma de Mallorca, Valencia, Gerona y Las. Palmas. Contrariamente, sobresalen por sus disminuciones de cuota. de mercado, Badajoz, Córdoba y Jaén, de una parte, y de otra, Bilbao, San Sebastián y Zaragoza.

Se perfila, pues, una tendencia hacia un mayor nivel de desarrollo de Cataluña y Baleares, Valencia-en sus tres Provincias-y Canarias, con una paralización o freno en su ritmo de crecimiento por lo que al País Vasco y a Aragón se refiere.

Por lo que a las Areas Comerciales hace referencia, aparecen con incremento de su cuota de mercado 41 Areas, y con disminución, 60 . En líneas generales, unas y otras configuran las Regiones ya descritas con respecto a las Zonas, aunque con algunas excepciones. Entre los incrementos, se mantiene en el Norte toda Galicia, con Asturias -excepto Oviedo-, Santander y Vitoria-sin Miranda de Ebro-; en el litoral mediterráneo oriental, Cataluña-con la excepción de Tortosa, Vich y Valls-, Baleares-sin Mahón-y la Región valenciana-sin Játiva y Utiel-; en el centro, Valladolid y Madrid, y en el Sur, Almería, Málaga-sin Antequera y Ronda-, Huelva-sin Ayamonte, aunque con Algeciras-y además Tenerife-sin Las Palmas-.

Entre las disminuciones de cuota de mercado resultan afectadas: las Areas del País Vasco, con Oviedo y Ponferrada y la excepción de Vitoria, y las de Navarra, Aragón y Logroño; el Valle del Duero, excepto León, con la adición de Miranda de Ebro y la excepción de Valladolid; la Submeseta Sur, con la excepción de Madrid; en Cataluña, Tortosa, Valls y Vich; en Baleares, Mahón; Murcia y Andalucía, sin Almería, Huelva y Málaga, pero con la incorporación de Ayamonte y la segregación de Algeciras, y en Canarias, la isla de La Palma. 
Destacan, una vez más, por la importancia relativa de sus incrementos, las Areas de Barcelona, Alicante, Valencia, Palma de Mallorca y Gerona, en el litoral mediterráneo oriental, y también Las Palmas; y por sus disminuciones, Bilbao, San Sebastián y Zaragoza, al Norte, y Badajoz, Sevilla, Córdoba y Jaén, al Sur.

\section{LA CUOTA DE MERCADO PER CÁPITA Y SU EVOLUCIÓN}

Parece conveniente considerar, por un momento, la cuota de mercado per cápita en 1979 y su evolución en el período considerado. En ambos casos nos referimos solamente a Areas Comerciales.

Considerando a la media nacional igual a la unidad, sólo es superada por 32 Areas de las 101 existentes. De ellas, diez se hallan en el Norte: Gijón, Bilbao, San Sebastián, Vitoria, Miranda de Ebro, Jaca, Pamplona, Logroño, Huesca y Zaragoza. En el litorial mediterráneo oriental se encuentran la mayor parte, nada menos que 17, y son: en Cataluña: en Gerona, Figueras, Olot y Puigcerdá; en Barcelona, Vich y Villafranca del Penedés; Lérida; en Tarragona, Reus y Valls; en Baleares, Palma de Mallorca, Ibiza, Ciudadela y Mahón, y Valencia y Alicante, en la Región valenciana. En la Meseta, Burgos, Palencia y Valladolid, en el Valle del Duero, y Madrid, en la Submeseta Sur. Y queda solamente aislada Arrecife.

Estas Areas resultan ser las más vigorosas en su desarrollo, en la mayor parte de los casos, fruto de un proceso largo en el tiempo, mientras que en otros obedece a circunstancias muy peculiares y recientes, como el caso de Arrecife.

En cuanto a la evolución de la renta per cápita en el período de referencia, anotemos que la han incrementado 61 Areas, la han visto disminuir 35 y en cinco permanece igual.

Las Areas han incrementado su renta per cápita bien por aumento de su cuota de mercado, manteniendo equilibrada su población o incluso registrándose un aumento moderado de ella, o bien por disminución de la población, ya que se trata de un cociente en que la disminución del divisor puede dar lugar a un mayor cociente, pero que el incremento del dividendo no siempre representa un cociente alto. Por ello se dan las circunstancias más diversas. De una parte, Areas con una alta cuota de mercado, pero con una gran población, presentan una cuota de mercado per cápita más bien débil, y otras con cuota de mercado baja registran, sin embargo, una cuota de mercado per cápita alta y aun creciente. 
Las Areas cuya "cuota de mercado per cápita» se ha incrementado en el período considerado se extienden por todo el territorio nacional. Al Norte, las ocho gallegas, Oviedo-salvo Avilés y Gijóny Santander. Todo el Valle del Duero, salvo Segovia, que se mantiene estacionaria. Toda la Submeseta Sur, a excepción de las Areas de Madrid y Toledo y la Provincia de Badajoz, si bien teniendo en cuenta que Mérida se mantiene estacionaria. En el Valle del Ebro, alto y medio, Miranda de Ebro, Logroño, Huesca, Barbastro y Teruel. Toda Cataluña, a excepción de las Areas de Barcelona, Tarragona y Tortosa. En Baleares solamente Ibiza. Las Provincias de Castellón y Alicante, y las Areas de Onteniente, Gandía y Utiel, las dos últimas estacionarias, en la de Valencia. Cartagena, en la Provincia de Murcia. En Andalucía, las Areas de Almería, Málaga, Sevilla, Huelva, Algeciras y Granada-estacionaria-. En Canarias, Santa Cruz de la Palma y la Provincia de Las Palmas.

Las 35 Areas que han visto disminuida su «cuota de mercado per cápita" se distribuyen en esta forma: Al Norte, además de Avilés y Gijón, las tres Provincias del País Vasco, con prolongación a Pamplona, Jaca, Zaragoza y Calatayud. En Cataluña: Barcelona, Tarragona y Tortosa. Toda Baleares, excepto Ibiza. En Levante, Valencia y Játiva, y en Murcia, la capital y Lorca. En la Meseta, Madrid, Toledo y la Provincia de Badajoz, excepto Mérida. En Andalucía, las Provincias de Jaén, Córdoba y Cádiz, y las Areas de Motril, Antequera, Ronda y Ayamonte. Y en Canarias, Santa Cruz de Tenerife.

Como se observa, mientras casi todas las Areas de la Meseta, con baja cuota de mercado, presentan, sin embargo, incrementos de su cuota de mercado per cápita en el período de referencia, la mayor parte de las Areas, cuya cuota de mercado per cápita disminuye, se sitúan en las mismas zonas periféricas, de predominio de las altas cuotas de mercado.

\section{LA CUOTA DE RIQUEZA ACTIVA DE LAS ZONAS $Y$ DE LAS AREAS COMERCIALES}

Por riqueza activa se entiende el conjunto de elementos que en la actividad económica del país contribuyen al proceso de desarrollo y a la formación de la renta nacional. Aunque estos elementos son inicialmente heterogéneos y de difícil medición en unidades monetarias, se han buscado expresiones homogéneas representati- 
vas de ellos y, consiguientemente, de la riqueza activa. Estas expresiones homogéneas son los índices demográfico, cultural y económico. Sobre la base de ellos se obtiene la cuota de riqueza activa, que se distribuye como la cuota de mercado proporcionalmente por Zonas y Areas sobre una total nacional de 100.000 unidades. Aunque la información contenida en el Anuario del Mercado Español se refiere solamente a Areas, hemos obtenido los valores correspondientes a las Zonas Comerciales también, y se presentan unos y otros valores en la tabla II del Apéndice Estadístico.

\section{El ANDICE DEMOGRAFICO}

Se elabora tomando como base la población total según el $\mathrm{Pa}$ drón Municipal de Habitantes renovado en 1975, la población urbana en la misma fecha y los inmigrantes recibidos en el año a nivel de Area Comercial, ante la imposibilidad de calcularlos a nivel municipal. De esta forma se expresa la importancia de la población, su distribución sobre el territorio y la fuerza atractiva de sus núcleos.

En orden a este índice sobresalen, en primer lugar, las Zonas Comerciales de Madrid y Barcelona, las dos principales urbes españolas, seguidas a cierta distancia de Valencia, Sevilla y Bilbao, las que, en conjunto, absorben el 41 por $100 \mathrm{del}$ total. Con un índice superior al 2 por 100 figuran otras diez Zonas, con el 24 por 100 en conjunto; con más del 1 por 100 figuran, a su vez, 16 Zonas, con el 22 por 100 en conjunto. Quedan 21 Zonas con índice inferior al 1 por 1.000 , que representan el 13 por 100 del total. Las 31 Zonas con índice superior al 1 por 100 configuran las cuatro grandes Regiones, que hemos descrito al tratar de la cuota de mercado. Al Norte, se hallan situadas La Coruña, Pontevedra, Oviedo, Santander, Bilbao, San Sebastián, Vitoria, Pamplona y Zaragoza. En el litoral mediterráneo oriental, Gerona, Barcelona, Tarragona, Castellón, Valencia, Alicante, Murcia y las Baleares. En la Meseta, León, Salamanca y Valladolid, en el Valle del Duero, y Madrid, Ciudad Real y Badajoz, en la Submeseta Sur. En el Sur, Jaén, Granada, Córdoba, Málaga, Sevilla y Cádiz, y en Canarias, Las Palmas y Santa Cruz de Tenerife.

Por lo que a las Areas hace referencia, la distribución es muy similar. Destacan también, en primer término, las de Madrid, Barcelona, Valencia, Sevilla y Bilbao, con 30 por 100 del índice en su conjunto, seguidas de seis Areas con índice superior al 2 por 100 
y un valor total del 13 por 100 , y 11 de índice superior al 1 por 100 , con un total del 14 por 100, quedando 79 Areas con indices inferiores al 1 por 100 , que absorben el 43 por 100 del total del índice. La distribución de estas Areas es similar a la descrita para las Zonas en sus cuatro Regiones.

\section{El INDICE CULTURAL}

Se obtiene tomando como base el número de alumnos de Bachillerato, incluidos los de los cursos quinto a octavo de Educación General Básica, y el número de librerías, comprendidas las editoriales, las librerías de nuevo y usado y los puestos fijos o quioscos que se dedican a la venta de libros. Es un índice significativo en cuanto representa un grado ideal de nivel cultural, básico para la formación técnica y universitaria.

También ocupan los primeros lugares, en cuanto a este índice, las Zonas de Madrid y Barcelona, seguidas de Valencia, Bilbao, Sevilla y, además, Oviedo, con el 42 por 100 del total. Les siguen ocho Zonas con más del 2 por 100 y 15 por encima del 1 por 100 , que representan, en conjunto, el 39 por 100 . Quedan 29 Zonas con índice inferior al 1 por 100, que suponen, en conjunto, el 19 por 100 del índice.

También destacan, en primer término, en cuanto a las Areas, las de Madrid, Barcelona, Valencia, Bilbao y Sevilla, con el 41 por 100. Figuran sólo dos Zonas con más del 2 por 100 y 15 con más del 1 por 100 , que suman, en conjunto, un 27 por 100 del índice, y quedan nada menos que 79 con índice inferior al 1 por 100 , con un conjunto del 32 por 100 del total del índice.

La distribución por Regiones es muy similar a la descrita con anterioridad.

\section{El ÍNDICE ECONÓMICO}

Se obtiene de manera más compleja. Se basa en los indicadores de riqueza agrícola, riqueza urbana y comercial e industrial que permiten obtener, en conjunto, el indicador general de riqueza. Con este indicador y las cifras de camiones y de teléfonos en servicio se obtiene el índice económico. Como en la tabla II se consigna solamente el índice económico y no se detallan sus componentes, los comentaremos someramente aquí, siempre refiriéndonos solamente a Areas Comerciales. 
El indicador de riqueza agricola difiere sustancialmente de las distribuciones contempladas hasta ahora. En él ocupan los primeros lugares las Areas de Sevilla y Córdoba, Valencia, Madrid, Lérida, Zaragoza y Valladolid, enumeradas en orden de importancia, y suman un 31 por 100 del índice total. Les siguen cinco Areas con índice superior al 2 por 100 , con un total equivalente al 11 por 100 , y 19 con índice superior al 1 por 100 , que representan un total del 26 por 100. $Y$ quedan 70 Areas con índice inferior al 1 por 100 , con un valor conjunto del 32 por 100. Las 31 Areas con índice superior al 1 por ciento configuran estas Regiones: El Norte comprende sólo Oviedo y Santander. Una segunda Región nórdica, siguiendo el criterio utilizado hasta el presente, está formada por Navarra y Aragón -Pamplona, Zaragoza, Huesca y Teruel-. En la Meseta destacan, en el Valle del Duero, León, Salamanca, Valladolid, Palencia y Soria, y en la Submeseta Sur, Madrid, Talavera, Ciudad Real, Cuenca, Badajoz, Cáceres y Albacete. En el litoral mediterráneo oriental, Lérida, Tortosa, Castellón, Valencia, Játiva y Murcia. Y al Sur, Sevilla, Huelva, Córdoba, Granada, Jaén y Ubeda. Y en Canarias, Las Palmas. Todas ellas con un sentido lógico de predominio agrario.

El indicador de riqueza urbana presenta una mayor similitud con el índice demográfico, aunque mucho más restringido, ya que, aunque se sitúan en primer lugar Barcelona y Madrid, Valencia, Bilbao, Málaga y Palma de Mallorca, con un peso conjunto del 44 por 100 del índice, sólo seis Areas destacan además por encima del 2 por 100 y 11 por encima del 1 por 100 del índice, con un total conjunto del 24 por 100 del índice total, quedando nada menos que 83 Areas con índice inferior al 1 por 100, que representa el 32 por 100 del índice. Las 23 Areas de referencia, con índice superior al 1 por 100, se distribuyen así: Al Norte, Vigo, Santander, Bilbao, San Sebastián, Pamplona y Zaragoza. Al Este, Gerona, Barcelona, Castellón, Valencia, Alicante, Murcia y Palma de Mallorca. En el Centro, solamente Madrid. Y al Sur, Málaga, Sevilla y Cádiz, y Las Palmas y Santa Cruz de Tenerife en las islas Canarias.

El indicador comercial $e$ industrial es muy significativo. Destacan también, en primer término, Barcelona, Madrid, Valencia y Bilbao, con el 34 por 100 del indicador. Les siguen cinco Areas con más del 2 por 100 y 22 con más del 1 por 100, que representan, en conjunto, el 34 por 100 del indicador, quedando 72 Areas por debajo del 1 por 100 , que, en conjunto, suman el 32 por 100 restante. Las 31 Areas con índice superior al 1 por 100 se agrupan así: Al 
Norte, La Coruña, Orense y Vigo, en Galicia; Oviedo y Avilés, en Asturias; seguidas, en orden geográfico, por Santander, Bilbao, San Sebastián, Pamplona y Zaragoza. Al Este, Lérida, Gerona, Barcelona, Tarragona y Reus, en Cataluña; Castellón, Valencia y Alicante, en la Región valenciana, además de Cartagena y Palma de Mallorca. En el Centro, Valladolid y Salamanca, en el Valle del Duero, y Madrid y Puertollano, en la Submeseta Sur. Y al Sur, Córdoba, Sevilla, Málaga y Huelva. Y en Canarias, Las Palmas y Santa Cruz de Tenerife.

Con los tres indicadores descritos se obtiene el indicador general conjunto de riqueza, en el que siguen destacando Barcelona y Madrid, Valencia y Bilbao, con el 37 por 100 del indicador, seguidas de seis Areas con más del 2 por 100 y 13 con más del 1 por 100, que suponen, en conjunto, el 33 por 100 del indicador. Y quedan 78 Areas con menos del 1 por 100, que disponen del 30 por $100 \mathrm{del}$ indicador. La distribución por Areas es similar a la descrita con referencia al indicador comercial e industrial, si prescindimos de Orense y Avilés, Lérida, Tarragona, Reus y Cartagena.

El índice económico, compuesto por el indicador general de riqueza, más los teléfonos y camiones, ofrece una visión de las Zonas Comerciales, a cuya cabeza figuran Barcelona y Madrid, Valencia, Bilbao, Sevilla y Alicante, con el 41 por 100 del índice total, seguidas de ocho Zonas por encima del 2 por 100 cada una, y 13 con más del 1 por 100, que, en conjunto, representan un 38 por 100; y quedan por debajo del 1 por 100, 25 Zonas con el 20 por 100. Estas 27 Zonas se distribuyen en las cuatro Regiones clásicas, consideradas en esta forma: En el Norte, La Coruña, Pontevedra, Oviedo, Santander, Bilbao, San Sebastián, Pamplona y Zaragoza. Al Este, Lérida, Gerona, Barcelona, Tarragona, Castellón, Valencia, Alicante y Murcia. En el Centro, Valladolid, León y Madrid. Al Sur, Granada, Córdoba, Málaga, Sevilla y Cádiz. Y en Canarias, Santa Cruz de Tenerife y Las Palmas.

El detalle por Areas es bastante similar, aunque no figura Alicante en el grupo principal que, en conjunto, representa el 41 por ciento del índice total, seguidas de cinco Areas por encima del 2 por 100 del índice, y 14 con más del 1 por 100, que suponen, en conjunto, el 30 por 100 . Y quedan con índice inferior al 1 por 100, 77 Areas, que suman el 29 por 100 del índice total. La distribución por Areas es similar a la de Zonas, si sustituimos a Pontevedra por Vigo y prescindimos de León, Tarragona y Cádiz. 


\section{LA CUOTA DE RIQUEZA ACTIVA}

Referida a un total de 100.000 unidades de los tres índices - demográfico, cultural y económico-, a los que viene a resumir, presenta una distribución, cuyo primer lugar ocupan Madrid y Barcelona, con el 29 por 100 del total. En segundo término figuran Valencia, Sevilla y Bilbao, con una cuota individual de entre el 3 y el 6 por 100 y un valor conjunto del 13 por 100. Con un índice entre el 2 y el 3 por 100 figuran La Coruña, Oviedo, Zaragoza, Alicante, Murcia, Málaga, Cádiz, Palma de Mallorca y Las Palmas, con el 22 por 100 en conjunto. Y entre el 1 y el 2 por 100 se hallan Pontevedra, León, Santander, San Sebastián, Vitoria, Pamplona, Lérida, Gerona, Tarragona, Valladolid, Castellón, Ciudad Real, Badajoz, Córdoba, Jaén, Granada y Santa Cruz de Tenerife, con un valor conjunto del 24 por 100 . Quedan 21 Zonas con un índice inferior al 1 por 100, que representan el 12 por100 del total. La distribución geográfica en las cuatro Regiones consideradas es similar a la establecida con referencia al índice económico.

En cuanto a las Areas, también figuran en primer término Madrid y Barcelona, con el 28 por 100, seguidas de Valencia, Sevilla y Bilbao, con el 12. Hay otras dos Areas entre el 2 y el 3 por 100 , 15 entre el 1 y el 2 por 100 , con un valor conjunto del 27 por 100 . Y quedan 77 Areas con el 33 por 100 restante. La distribución geográfica es similar a la de las Zonas, si se prescinde de Pontevedra y Vitoria, Lérida y Tarragona, León, Ciudad Real y Badajoz, y Cádiz y Jaén, con lo que la expansión en Areas resulta algo más reducida que la de Zonas, en sus mismos niveles.

\section{EL INDICE TURISTICO Y EL NIVEL DE DESARROLLO}

Aunque el índice turístico no figura como uno de los componentes de la cuota de riqueza activa, se ha elaborado también teniendo en cuenta las plazas en hostelería y campings, sus precios y tiempo en que están abiertos al año. De su examen reducido a Areas Comerciales, se deduce que ocupa el primer lugar Palma de Mallorca, con el 16 por 100 del total, y a ella siguen Madrid, con el 11; Barcelona y Málaga, con el 8; Las Palmas y Santa Cruz de Tenerife, con el 6; Alicante, con el 5; Gerona, con el 4; Ibiza y Sevilla, con el 3; Valencia, con el 2, y Lérida y Granada, con el 1, de 
forma que 13 Areas absorben el 74 por 100 del índice. Las 88 Areas restantes se reparten el 26 por 100 restante.

El nivel de desarrollo es la relación existente entre la cuota de riqueza activa de cada Area y el índice de su población total, considerando 100 el nivel medio nacional. Estos niveles se sitúan por encima o debajo del nivel medio nacional, y expresan el nivel de desarollo de cada Area. Son 41 las Areas que ofrecen un nivel superior al promedio nacional, de las que pudiéramos decir que tienen un porvenir más claro en el orden socioeconómico. Las enumeramos a continuación, según las cuatro Regiones clásicas que venimos considerando.

Al Norte se hallan: Gijón y Avilés; las tres Provincias del País Vasco, con Miranda de Ebro, Pamplona y las tres Areas de la Provincia de Huesca. Al Este se sitúan Gerona, Figueras, Puigcerdá y Olot, Barcelona, Tarragona, Reus y Valls; Castellón, Valencia, Alicante, Alcoy, Elche y Cartagena; y Palma de Mallorca, Ibiza, Mahón y Ciudadela. En el Centro, Burgos, Palencia, Valladolid y Segovia, en el Valle del Duero, y Madrid, Guadalajara, Toledo y Puertollano, en la Submeseta Sur. Al Sur, Málaga, Algeciras y La Línea, y Arrecife y Las Palmas, en Canarias.

\section{APENDICE ESTADISTICO}

1. Evolución de las cuotas de MERCado en LaS ZoNaS Y eN LAS AREAS CoMERCIALES DURANTE EL PERfodo 1967-1979

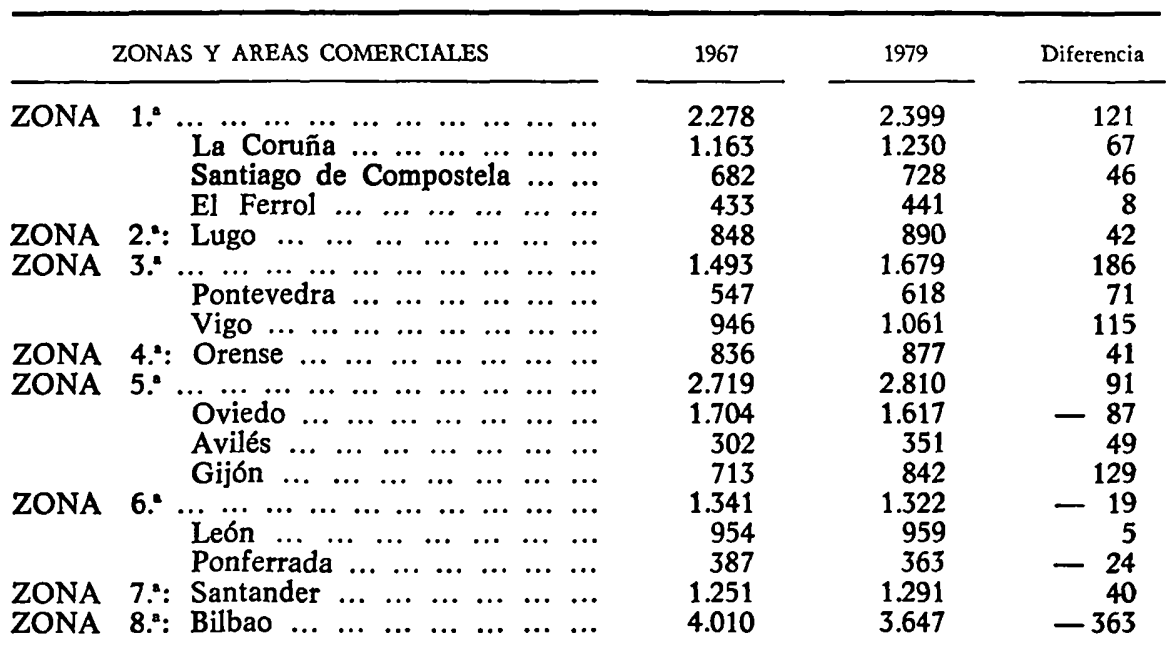




\begin{tabular}{|c|c|c|c|c|}
\hline \multicolumn{2}{|c|}{ ZONAS Y AREAS COMERCIALES } & 1967 & 1979 & Diferencia \\
\hline $\begin{array}{l}\text { ZONA 9.:: } \\
\text { ZONA 10:" }\end{array}$ & 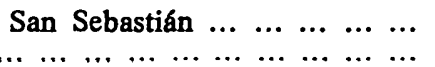 & $\begin{array}{r}2.298 \\
767\end{array}$ & $\begin{array}{r}1.935 \\
889\end{array}$ & $\begin{array}{r}-363 \\
122\end{array}$ \\
\hline & 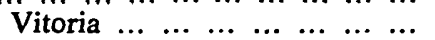 & 623 & 756 & 135 \\
\hline & Miranda de Ebro $\ldots \ldots \ldots$ & 144 & 133 & -11 \\
\hline ZONA 11: & 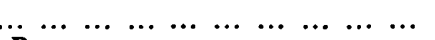 & 805 & 771 & -34 \\
\hline & $\begin{array}{llllllll}\text { Burgos } & \ldots & \ldots & \ldots & \ldots & \ldots & \ldots & \ldots\end{array}$ & 657 & 644 & -13 \\
\hline & Aranda de Duero $\ldots \ldots \ldots \ldots$ & 148 & 127 & -21 \\
\hline ZONA 12.. & $\begin{array}{lllllll}\text { Logroño } & \ldots & \ldots & \ldots & \ldots & \ldots & \ldots\end{array}$ & 940 & 876 & -64 \\
\hline ZONA $13 . "$ & $\begin{array}{lllllll}\text { Pamplona } & \ldots & \ldots & \ldots & \ldots & \ldots & \ldots\end{array}$ & 1.463 & 1.397 & -66 \\
\hline & $\begin{array}{lllllllllll} & \ldots & \ldots & \ldots & \cdots & \cdots & \ldots & \ldots & \ldots & \cdots\end{array}$ & 502 & 443 & -59 \\
\hline & $\begin{array}{llllllll}\text { Huesca } & \ldots & \ldots & \ldots & \ldots & \ldots & \ldots & \cdots\end{array}$ & 202 & 195 & -7 \\
\hline & $\begin{array}{llllllll}\text { Barbastro } & \cdots & \cdots & \ldots & \cdots & \cdots & \cdots \\
\text { Jaca } & \ldots & \ldots & \ldots & \ldots & \ldots & \ldots & \ldots\end{array}$ & $\begin{array}{l}183 \\
117\end{array}$ & $\begin{array}{r}157 \\
91\end{array}$ & \\
\hline ZONA 15." & 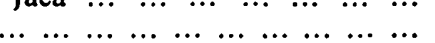 & 1.229 & 1.302 & -73 \\
\hline & $\begin{array}{llllllll} & \\
\text { Lérida } & \ldots & \ldots & \ldots & \ldots & \ldots & \ldots & \ldots\end{array}$ & 1.181 & 1.249 & 68 \\
\hline & $\begin{array}{lllllll}\text { Puigcerdá } & \ldots & \ldots & \ldots & \ldots & \ldots & \ldots\end{array}$ & 48 & 53 & 5 \\
\hline ZONA 16:" & $\begin{array}{llllllllll}\ldots & \ldots & \ldots & \ldots & \ldots & \ldots & \ldots & \ldots & \ldots & \ldots\end{array}$ & 1.787 & 2.099 & 312 \\
\hline & $\begin{array}{llllllll}\text { Gerona } & \ldots & \ldots & \ldots & \ldots & \ldots & \ldots & \ldots\end{array}$ & 1.049 & 1.266 & 217 \\
\hline & $\begin{array}{llllllll}\text { Figueras } & \ldots & \ldots & \ldots & \ldots & \ldots & \ldots\end{array}$ & 243 & 318 & 75 \\
\hline & 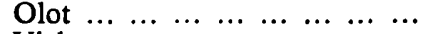 & 130 & 151 & 21 \\
\hline & $\begin{array}{llllllllll} & V i c h & \ldots & \ldots & \ldots & \ldots & \ldots & \ldots & \ldots & \ldots\end{array}$ & 365 & 364 & - \\
\hline ZONA 17." & 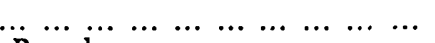 & 14.708 & 15.370 & 662 \\
\hline & $\begin{array}{llllllll}\text { Barcelona } & \ldots & \ldots & \ldots & \ldots & \ldots & \ldots\end{array}$ & 13.964 & 14.528 & 564 \\
\hline & $\begin{array}{lllllllll}\text { Manresa } & \ldots & \ldots & \ldots & \ldots & \ldots & \ldots & \ldots\end{array}$ & 518 & 555 & 37 \\
\hline & Vilafranca del Penedés $\ldots . .$. & 226 & 287 & 61 \\
\hline ZONA $18 .^{\circledR}$ & $\begin{array}{llllllllll} & \ldots & \ldots & \ldots & \ldots & \ldots & \ldots & \ldots & \ldots & \ldots\end{array}$ & 931 & 1.095 & 164 \\
\hline & $\begin{array}{lllllll}\text { Tarragona } & \ldots & \ldots & \ldots & \ldots & \ldots & \ldots\end{array}$ & 340 & 475 & 135 \\
\hline & $\begin{array}{lllllllll}\text { Reus } & \ldots & \ldots & \ldots & \ldots & \ldots & \ldots & \ldots & \ldots\end{array}$ & 481 & 515 & 34 \\
\hline & $\begin{array}{llllllll}\text { Valls } & \ldots & \ldots & \ldots & \ldots & \ldots & \ldots & \ldots\end{array}$ & 110 & 105 & - \\
\hline ZONA 19.": & $\begin{array}{ccccccc}\text { Tortosa } & \ldots & \ldots & \ldots & \ldots & \ldots & \ldots\end{array}$ & 450 & 382 & -68 \\
\hline ZONA 20." & 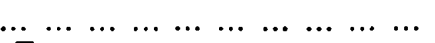 & 2.820 & 2.493 & -327 \\
\hline & $\begin{array}{lllllll}\text { Zaragoza } & \ldots & \ldots & \ldots & \ldots & \ldots & \ldots\end{array}$ & 2.632 & 2.368 & -264 \\
\hline & $\begin{array}{lllllll}\text { Calatayud } & \ldots & \ldots & \ldots & \ldots & \ldots & \ldots\end{array}$ & 188 & 125 & -63 \\
\hline ZONA 21.": & 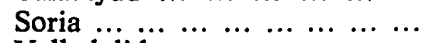 & 280 & 209 & -71 \\
\hline ZONA 22.": & $\begin{array}{lllllll}\text { Valladolid } & \ldots & \ldots & \ldots & \ldots & \ldots & \ldots\end{array}$ & 1.178 & 1.289 & 111 \\
\hline ZON & $\begin{array}{lllllll}\text { Palencia } & \ldots & \ldots & \ldots & \ldots & \ldots & \ldots\end{array}$ & 627 & 524 & -103 \\
\hline ZONA 24:: & $\begin{array}{lllllllll}\text { Zamora } & \ldots & \ldots & \ldots & \ldots & \ldots & \ldots & \ldots\end{array}$ & 557 & 483 & -74 \\
\hline $25 .^{\circ}$ & $\begin{array}{llllllllllll} & \ldots & \ldots & \ldots & \ldots & \ldots & \ldots & \ldots & \ldots & \ldots & \ldots\end{array}$ & 1.044 & 959 & -85 \\
\hline & $\begin{array}{lllllll}\text { Salamanca } & \ldots & \ldots & \ldots & \ldots & \ldots & \ldots\end{array}$ & 849 & 799 & -50 \\
\hline & $\begin{array}{llllllll}\text { Béjar } & \ldots & \ldots & \ldots & \ldots & \ldots & \ldots & \ldots\end{array}$ & 82 & 70 & -12 \\
\hline & $\begin{array}{llllll}\text { Ciudad Rodrigo } & \ldots & \ldots & \ldots & \ldots\end{array}$ & 113 & 90 & -23 \\
\hline ZONA 26.: & $\begin{array}{llllllll}\operatorname{Segovia} & \ldots & \ldots & \ldots & \ldots & \ldots & \ldots & \ldots\end{array}$ & 405 & 315 & -90 \\
\hline ZONA 27.": & 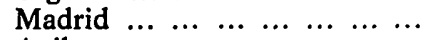 & 15.569 & 15.726 & 157 \\
\hline ZONA 28.": & $\begin{array}{llllllll}\text { Avila } & \ldots & \ldots & \ldots & \ldots & \ldots & \ldots & \ldots\end{array}$ & 338 & 282 & -56 \\
\hline ZONA 29.": & $\begin{array}{cccccc}\text { Guadalajara } & \ldots & \ldots & \ldots & \ldots & \ldots\end{array}$ & 318 & 288 & -30 \\
\hline ZONA 30.": & $\begin{array}{lllllllll}\text { Teruel } & \ldots & \ldots & \ldots & \ldots & \ldots & \ldots & \ldots\end{array}$ & 264 & 202 & -62 \\
\hline ZONA 31.:: & $\begin{array}{lllllll}\text { Castellón } & \ldots & \ldots & \ldots & \ldots & \ldots & \ldots\end{array}$ & 970 & 1.047 & 77 \\
\hline ZONA 32." & $\begin{array}{lllllllllll}\ldots & \ldots & \ldots & \ldots & \ldots & \ldots & \ldots & \ldots & \ldots & \ldots\end{array}$ & 5.826 & 6.169 & 343 \\
\hline & $\begin{array}{lllllll}\text { Valencia } & \ldots & \ldots & \ldots & \ldots & \ldots & \ldots\end{array}$ & 4.858 & 5.233 & 375 \\
\hline & 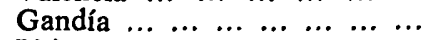 & 469 & 483 & 14 \\
\hline & $\begin{array}{llllllll}\text { Játiva } & \ldots & \ldots & \ldots & \ldots & \ldots & \ldots & \ldots\end{array}$ & 298 & 271 & -27 \\
\hline & 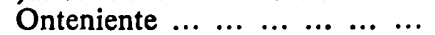 & 114 & 117 & \\
\hline & $\begin{array}{llllllll}\text { Utiel } & \ldots & \ldots & \ldots & \ldots & \ldots & \ldots & \ldots\end{array}$ & 87 & 65 & -22 \\
\hline ZONA 33.: & $\begin{array}{lllllllll}\text { Cuenca } & \ldots & \ldots & \ldots & \ldots & \ldots & \ldots & \ldots\end{array}$ & 306 & 240 & -66 \\
\hline ZONA 34.": & $\begin{array}{lllllllll}\text { Toledo } & \ldots & \ldots & \ldots & \ldots & \ldots & \ldots & \ldots\end{array}$ & 391 & 349 & -42 \\
\hline $70 N 35 \mathrm{a}$ & Talavera de la Reina ... ... ... & 520 & 505 & -15 \\
\hline
\end{tabular}




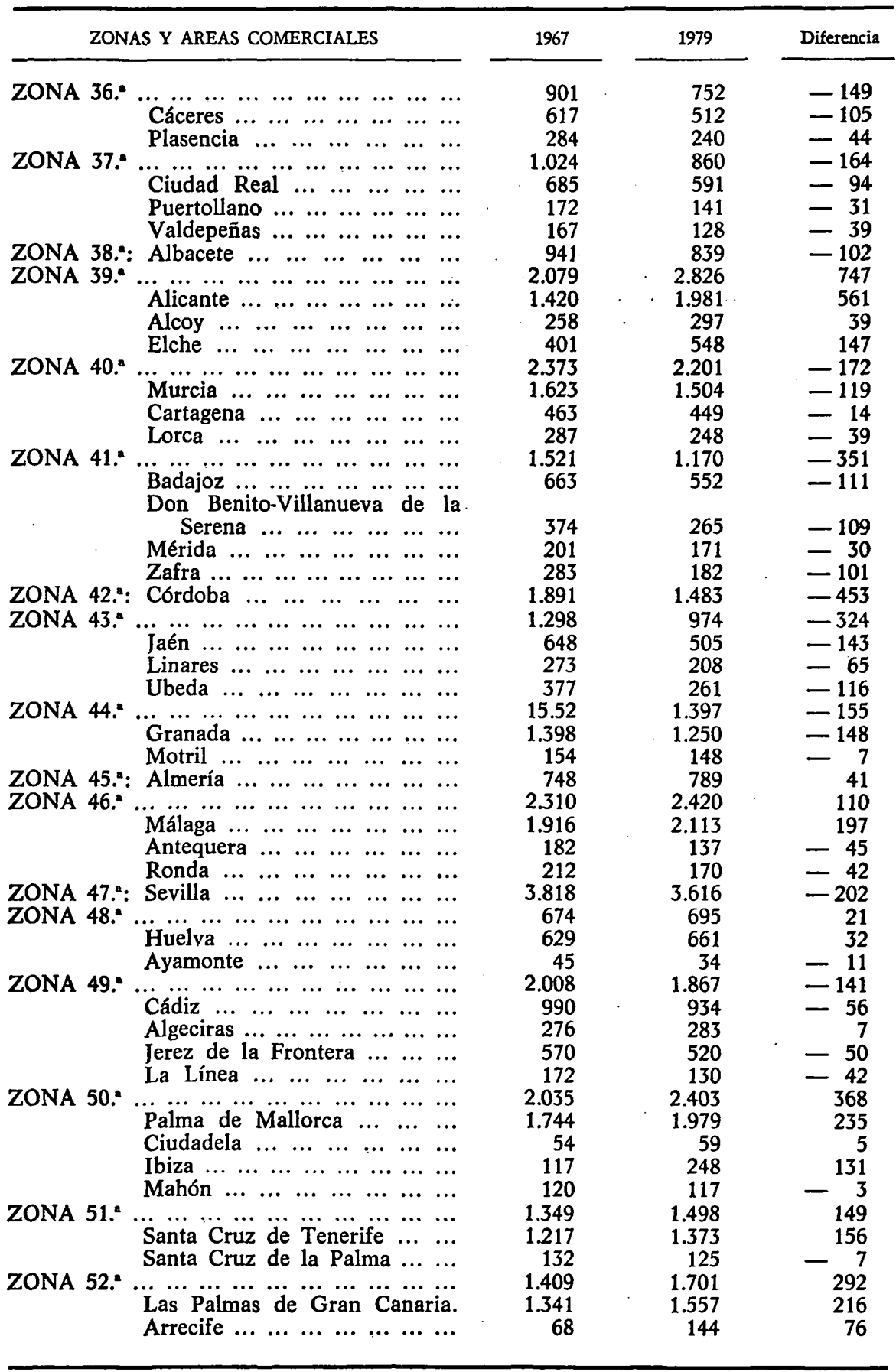


2. LOS ÍNDICES DEMOGRÁFICO, CULTURAL Y ECONÓMICO Y LA CUOTA DB

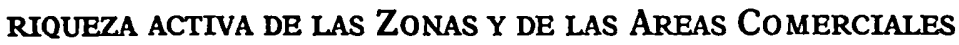

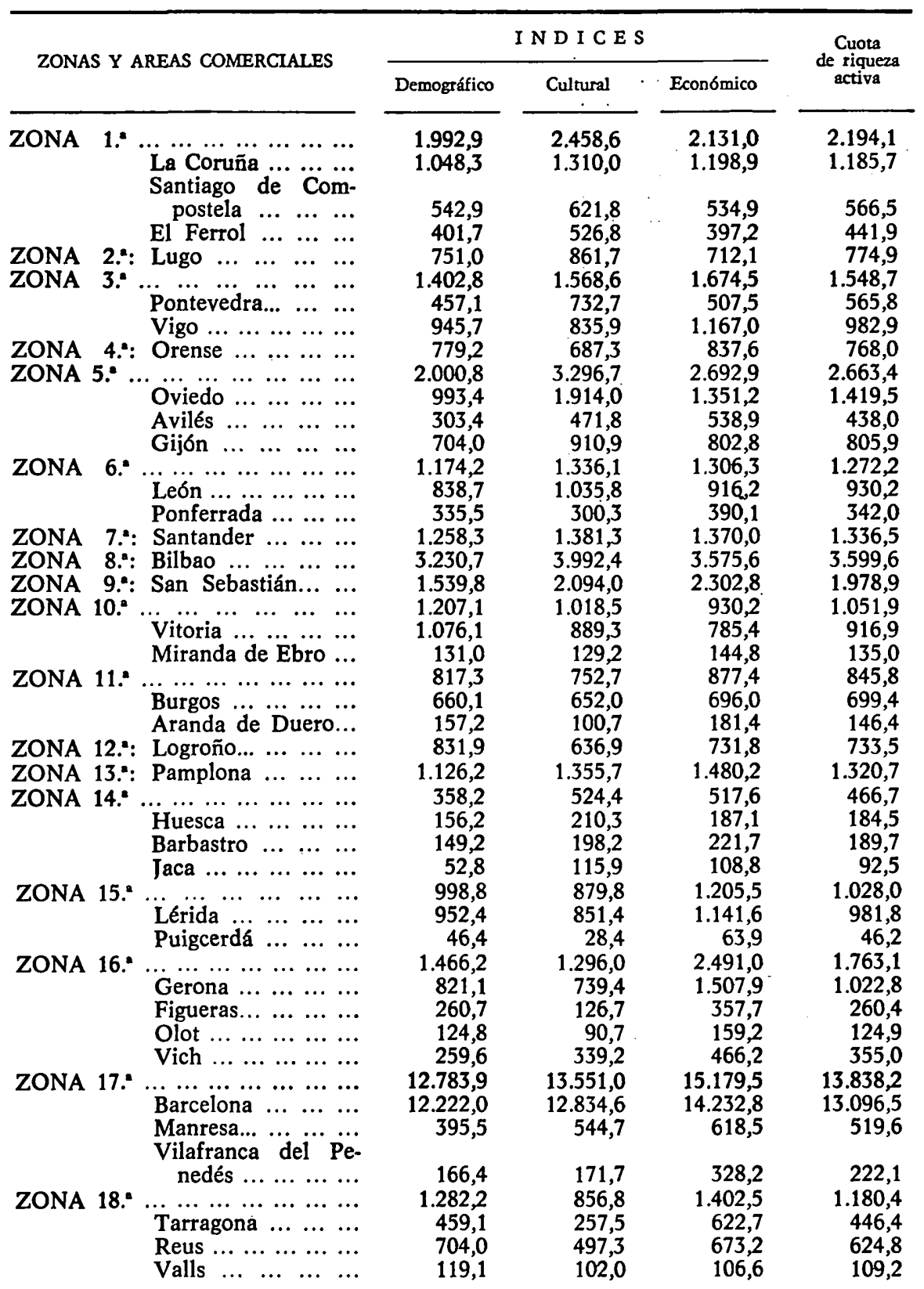




\begin{tabular}{|c|c|c|c|c|c|}
\hline \multirow{2}{*}{\multicolumn{2}{|c|}{ ZONAS Y AREAS COMERCIALES }} & \multicolumn{3}{|c|}{ I N D I C E S } & \multirow{2}{*}{$\begin{array}{l}\text { Cuota } \\
\text { de riqueza } \\
\text { activa }\end{array}$} \\
\hline & & Demográfico & Cultural & Económico & \\
\hline \multirow[t]{2}{*}{$\begin{array}{l}\text { ZONA 19.: } \\
\text { ZONA 20:" }\end{array}$} & 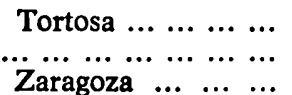 & $\begin{array}{r}402,8 \\
2.562,4 \\
2.436,5\end{array}$ & $\begin{array}{r}398,8 \\
2.215,1 \\
2.066,0\end{array}$ & $\begin{array}{r}427,4 \\
2.495,8 \\
2.385,3\end{array}$ & $\begin{array}{r}409,7 \\
2.424,6 \\
2.296,1\end{array}$ \\
\hline & 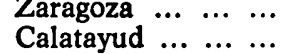 & $\begin{array}{r}2.436,5 \\
125,9\end{array}$ & $\begin{array}{r}2.006,0 \\
149,1\end{array}$ & $\begin{array}{r}2.50 J, 3 \\
110,5\end{array}$ & $\begin{array}{r}2.290,1 \\
128,5\end{array}$ \\
\hline \multirow{4}{*}{\multicolumn{2}{|c|}{ 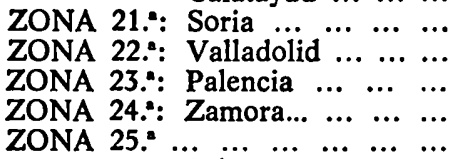 }} & 193,0 & 249,9 & 222,2 & 221,7 \\
\hline & & $1.574,9$ & $1.241,2$ & $1.088,5$ & $1.301,5$ \\
\hline & & 629,1 & 664,7 & 418,0 & 570,6 \\
\hline & & 485,7 & 474,1 & 456,4 & 472,1 \\
\hline \multirow{2}{*}{\multicolumn{2}{|c|}{ 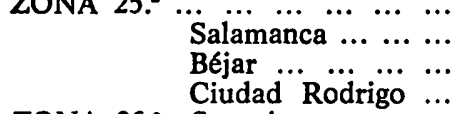 }} & $\begin{array}{r}1.109,8 \\
890,1\end{array}$ & $\begin{array}{r}1.007,8 \\
823,2\end{array}$ & $\begin{array}{l}768,8 \\
656,0\end{array}$ & $\begin{array}{l}962,2 \\
789,8\end{array}$ \\
\hline & & $\begin{array}{l}107,0 \\
112,7\end{array}$ & $\begin{array}{l}87,9 \\
96,7\end{array}$ & $\begin{array}{l}57,0 \\
55,8\end{array}$ & $\begin{array}{l}84,0 \\
88,4\end{array}$ \\
\hline \multirow{6}{*}{\multicolumn{2}{|c|}{ 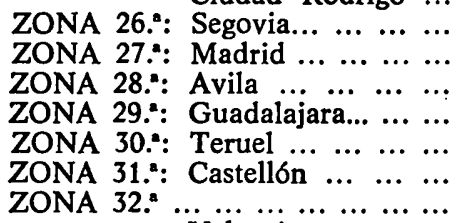 }} & 478,8 & 425,8 & 373,9 & 426,2 \\
\hline & & $14.715,2$ & $16.339,9$ & $14.691,0$ & $15.248,7$ \\
\hline & & 371,9 & 381,6 & 345,2 & 366,2 \\
\hline & & $\begin{array}{l}497,8 \\
2372\end{array}$ & 504,4 & 398,3 & $\begin{array}{l}466,8 \\
2199\end{array}$ \\
\hline & & $\begin{array}{r}237,2 \\
1.229,7\end{array}$ & $\begin{array}{l}201,1 \\
933,7\end{array}$ & $\begin{array}{r}221,3 \\
1.232,9\end{array}$ & $\begin{array}{r}219,9 \\
1.132,1\end{array}$ \\
\hline & & $5.784,7$ & $5.221,7$ & $6.329,5$ & $5.778,7$ \\
\hline \multirow{5}{*}{ LUINA 32} & $\begin{array}{llll}\text { Valencia } & \ldots & \ldots & \ldots\end{array}$ & $5.038,9$ & $4.247,6$ & $5.361,4$ & $4.882,6$ \\
\hline & Gandia $\ldots \ldots \ldots \ldots$ & 373,3 & 466,8 & 511,6 & 450,6 \\
\hline & $\begin{array}{lllll}\text { Játiva } & \ldots & \ldots & \ldots & \ldots\end{array}$ & 201,8 & 292,8 & 277,8 & 257,5 \\
\hline & Onteniente $\ldots . \ldots \ldots$ & 119,0 & 147,5 & 115,3 & 127,3 \\
\hline & Utiel $\ldots \ldots \ldots \ldots \ldots$ & 51,7 & 67,0 & 63,4 & 60,7 \\
\hline \multirow{4}{*}{\multicolumn{2}{|c|}{ 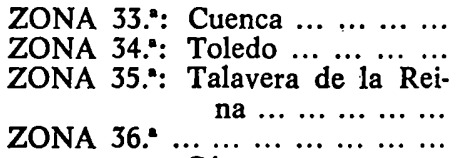 }} & 275,3 & 374,6 & 244,3 & 298,1 \\
\hline & & 366,7 & 483,1 & 354,5 & 401,4 \\
\hline & & 632,4 & 524,6 & 447,8 & 534,9 \\
\hline & & 980,7 & 767,5 & 592,5 & 780,2 \\
\hline \multirow{2}{*}{ ZONA 36." } & Cáceres......... & 637,2 & 545,0 & 434,9 & 539,0 \\
\hline & $\begin{array}{llll}\text { Plasencia } & \ldots & \ldots & \ldots\end{array}$ & 343,5 & 222,5 & 157,6 & 241,2 \\
\hline \multirow[t]{4}{*}{ ZONA $370^{\circ}$} & $\cdots, \cdots, \cdots \cdots \cdots$ & $1.334,9$ & $1.060,3$ & 931,4 & $1.108,9$ \\
\hline & Ciudad Real ... ... & 840,5 & 678,5 & 540,8 & 686,6 \\
\hline & Puertollano... ... ... & 288,5 & 196,2 & 256,0 & 246,9 \\
\hline & Valdepeñas... $\ldots \ldots \ldots$ & 205,9 & 185,6 & 134,6 & 175,4 \\
\hline \multirow{5}{*}{$\begin{array}{l}\text { ZONA 38.": } \\
\text { ZONA 39." }\end{array}$} & Albacete $\quad \ldots \quad \ldots \quad \ldots$ & 961,0 & 900,0 & 707,4 & 856,2 \\
\hline & $\begin{array}{llllllll} & \ldots & \ldots & \ldots & \ldots & \ldots & \ldots & \ldots\end{array}$ & $2.777,6$ & $2.565,5$ & $3.310,9$ & $2.884,7$ \\
\hline & Alicante $\ldots \ldots \ldots$ & $1.747,9$ & $1.622,7$ & $2.245,8$ & $1.872,1$ \\
\hline & $\begin{array}{llllll}\text { Alcoy } & \ldots & \ldots & \ldots & \ldots\end{array}$ & 301,2 & 570,0 & 319,7 & 397,0 \\
\hline & $\begin{array}{lllll}\text { Elche } & \ldots & \ldots & \ldots & \ldots\end{array}$ & 728,5 & 372,8 & 745,4 & 615,6 \\
\hline \multirow[t]{4}{*}{ ZONA 40." } & $\begin{array}{lllllllllll} & \cdots & \cdots & \ldots & \ldots & \ldots & \ldots & \ldots\end{array}$ & $2.262,7$ & $2.438,3$ & $2.826,5$ & $2.509,3$ \\
\hline & Murcia $\ldots \ldots \ldots \ldots$ & $1.394,4$ & $1.629,6$ & $1.714,8$ & $1.579,7$ \\
\hline & Cartagena $\ldots \ldots \ldots$ & 550,2 & 625,1 & 778,8 & 651,4 \\
\hline & Lorca $\ldots \ldots \ldots$ & 318,1 & 183,6 & 332,9 & 278,2 \\
\hline \multirow{5}{*}{ ZONA 41: } & 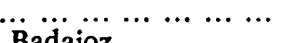 & $1.449,8$ & $1.481,2$ & $\begin{array}{l}877,9 \\
4128\end{array}$ & $1.269,6$ \\
\hline & 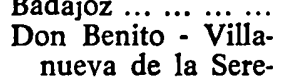 & & & 412,8 & \\
\hline & . $\ldots \ldots \ldots \ldots$ & 376,7 & 284,8 & 202,0 & 287,8 \\
\hline & Mérida ... ... ... ... & 213,2 & 220,1 & 137,7 & 190,3 \\
\hline & $\begin{array}{llllll}\text { Zafra } & \ldots & \ldots & \ldots & \ldots\end{array}$ & 212,5 & 178,6 & 125,4 & 172,2 \\
\hline \multirow{4}{*}{$\begin{array}{l}\text { ZONA 42.: } \\
\text { ZONA 43." }\end{array}$} & $\begin{array}{llll}\text { Córdoba } & \ldots & \ldots & \ldots\end{array}$ & $2.105,2$ & $1.667,0$ & $1.227,8$ & $1.666,7$ \\
\hline & $\because \cdots \cdots \cdots \cdots$ & $1.337,2$ & $1.455,0$ & 885,0 & $1.225,9$ \\
\hline & $\begin{array}{llllll}\text { Jaén } & \ldots & \ldots & \ldots & \ldots & \ldots\end{array}$ & 722,6 & 844,4 & 481,7 & 682,9 \\
\hline & $\begin{array}{lllll}\text { Linares } & \ldots & \ldots & \ldots & \ldots \\
\text { Ubeda } & \ldots & \ldots & \ldots & \ldots\end{array}$ & $\begin{array}{l}319,3 \\
295,1\end{array}$ & $\begin{array}{l}2 / 4,3 \\
336,6\end{array}$ & $\begin{array}{l}198,8 \\
204,5\end{array}$ & $\begin{array}{l}204,5 \\
278,7\end{array}$ \\
\hline
\end{tabular}




\begin{tabular}{|c|c|c|c|c|c|}
\hline \multirow{2}{*}{\multicolumn{2}{|c|}{ ZONAS Y AREAS COMERCIALES }} & \multicolumn{3}{|c|}{ I N D I C E S } & \multirow{2}{*}{$\begin{array}{l}\text { Cuota } \\
\text { de riqueza } \\
\text { activa }\end{array}$} \\
\hline & & Demográfico & Cultural & Económico & \\
\hline \multirow[t]{3}{*}{ ZONA 44: } & $\begin{array}{llll}. & \ldots & \ldots & \ldots\end{array}$ & $2.558,3$ & $1.850,4$ & $1.295,2$ & $1.901,3$ \\
\hline & Granada $\ldots \ldots \ldots$ & $2.374,5$ & $1.696,8$ & $1.131,0$ & $1.734,1$ \\
\hline & $\begin{array}{lllll}\text { Motril } \ldots & \ldots & \ldots & \ldots\end{array}$ & 183,8 & 153,6 & 164,2 & 167,2 \\
\hline \multirow{5}{*}{$\begin{array}{l}\text { ZONA } 45 .^{2}: \\
\text { ZONA } 46 .\end{array}$} & Almería... ... ... ... & 999,2 & 989,2 & 921,4 & 969,9 \\
\hline & $\begin{array}{llllll}\ldots & \ldots & \ldots & \ldots & \ldots & \ldots\end{array}$ & $2.737,2$ & $2.286,9$ & $2.610,3$ & $2.544,7$ \\
\hline & Málaga $\ldots \ldots \ldots \ldots$ & $2.255,2$ & $1.911,7$ & $2.361,2$ & $2.176,0$ \\
\hline & Antequera $\ldots \ldots \ldots$ & 210,2 & 201,2 & 123,9 & 178,4 \\
\hline & Ronda $\ldots \ldots \ldots c c$ & 271,8 & 174,0 & 125,2 & 190,3 \\
\hline \multirow{4}{*}{$\begin{array}{l}\text { ZONA } 47 .: \text { : } \\
\text { ZONA } 48 .:\end{array}$} & Sevilla $\ldots \ldots \ldots \ldots$ & $4.528,4$ & $3.663,5$ & $3.023,8$ & $3.738,6$ \\
\hline & $\begin{array}{llllllll} & \ldots & \cdots & \ldots & \ldots & \ldots & \ldots & \ldots\end{array}$ & 678,0 & 741,7 & 747,8 & 722,5 \\
\hline & Huelva $\ldots \ldots \ldots \ldots$ & 641,0 & 700,2 & 726,2 & 689,1 \\
\hline & Ayamonte $\ldots \ldots \ldots$ & 37,0 & 41,5 & 21,6 & 33,4 \\
\hline \multirow[t]{4}{*}{ ZONA 49." } & $\ldots \ldots \ldots \ldots$ & $2.608,0$ & $2.441,1$ & $1.873,3$ & $2.298,7$ \\
\hline & $\begin{array}{lllll}\text { Cádiz } & \ldots & \ldots & \ldots & \ldots\end{array}$ & $1.197,2$ & $1.143,1$ & 856,1 & $1.065,7$ \\
\hline & $\begin{array}{llll}\text { Algeciras } & \ldots & \ldots & \ldots\end{array}$ & 500,6 & 351,2 & 314,2 & 391,7 \\
\hline & $\begin{array}{l}\text { Jerez de la Frontera. } \\
\text { La Línea } \ldots \ldots \ldots\end{array}$ & $\begin{array}{l}647,2 \\
263,0\end{array}$ & $\begin{array}{l}719,9 \\
226,9\end{array}$ & $\begin{array}{l}492,3 \\
174,7\end{array}$ & $\begin{array}{l}619,8 \\
221,5\end{array}$ \\
\hline \multirow[t]{5}{*}{ ZONA 50: } & 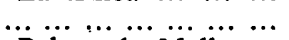 & $2.281,0$ & $1.644,8$ & $2.796,0$ & $2.240,6$ \\
\hline & Palma de Mallorca. & $2.002,1$ & $1.328,2$ & $2.251,3$ & $1.860,5$ \\
\hline & Ciudadela $\ldots \ldots \ldots$ & 61,8 & 90,5 & 80,6 & 77,6 \\
\hline & $\begin{array}{lllll}\text { Ibiza } & \ldots & \ldots & \ldots & \ldots\end{array}$ & 97,5 & 88,1 & 320,4 & 168,7 \\
\hline & Mahón $\ldots \ldots \ldots$..... & 119,6 & 138,0 & 143,7 & 133,8 \\
\hline \multirow[t]{3}{*}{ ZONA 51: } & 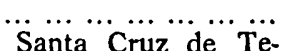 & $1.396,1$ & $1.774,0$ & $1.741,9$ & $1.637,4$ \\
\hline & \begin{tabular}{cccc}
\multicolumn{2}{c}{ nerife $\ldots$} & $\ldots \ldots$ & $\ldots$ \\
Santa & Cruz & de & la
\end{tabular} & $1.271,2$ & $1.538,9$ & $1.590,0$ & $1.466,8$ \\
\hline & $\begin{array}{llll}\text { Palma } & \ldots & \ldots & \ldots\end{array}$ & 124,9 & 235,1 & 151,9 & 170,6 \\
\hline \multirow[t]{3}{*}{ ZONA 52." } & 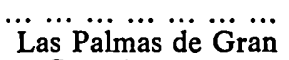 & $2.446,8$ & $2.045,6$ & $1.731,9$ & $2.074,8$ \\
\hline & Canaria $\ldots \ldots \ldots$ & $2.223,6$ & $1.897,1$ & $1.600,9$ & $1.907,2$ \\
\hline & Arrecife $\ldots \ldots \ldots \ldots$ & 223,2 & 148,5 & 131,0 & 167,6 \\
\hline
\end{tabular}


REVL-1981, núm. 211. BALLESTER ROS, IGNACIO. CARACTERISTICAS DE LAS CUOTAS DE ME... REVL-1981, núm. 211. BALLESTER ROS, IGNACIO. CARACTERISTICAS DE LAS CUOTAS DE ME... 


\section{REVISTA}

DE

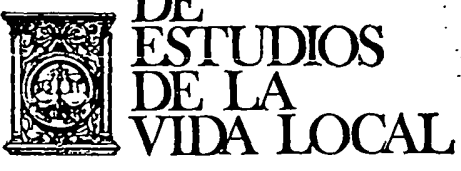

IV. JURISPRUDENCIA

REVL-1981, núm. 211. BALLESTER ROS, IGNACIO. CARACTERISTICAS DE LAS CUOTAS DE ME... 
REVL-1981, núm. 211. BALLESTER ROS, IGNACIO. CARACTERISTICAS DE LAS CUOTAS DE ME... REVL-1981, núm. 211. BALLESTER ROS, IGNACIO. CARACTERISTICAS DE LAS CUOTAS DE ME... 